\title{
Diagnosis of emphysema in patients with chronic bronchitis: a new approach
}

\author{
M. Kohlhäufl*, P. Brand**, T. Selzer**, G. Scheuch**, Th. Meyer**, N. Weber**, \\ H. Schulz**, K. Häussinger*, J. Heyder**
}

Diagnosis of emphysema in patients with chronic bronchitis: a new approach. M. Kohlhäufl, P. Brand, T. Selzer, G. Scheuch, Th. Meyer, N. Weber, H. Schulz, K. Häussinger, J. Heyder. CERS Journals Ltd 1998.

ABSTRACT: Aerosol-derived airway morphometry (ADAM) and aerosol bolus dispersion $(D)$ are altered in patients or animal models with lung emphysema. This study was performed to examine the sensitivity and specificity of ADAM and $D$ in the detection of emphysema in vivo compared with conventional lung function parameters.

The study comprised patients with chronic obstructive bronchitis (COB) without emphysema (group COB; $n=19$, age $56 \pm 8$ yrs, forced expiratory volume in one second (FEV1)/vital capacity (VC) $66 \pm 12 \%$ predicted) and patients with chronic bronchitis with high-resolution computed tomography-confirmed emphysema (group COB-E; $\mathrm{n}=20$, age $65 \pm 7 \mathrm{yrs}, \mathrm{FEV} 1 / \mathrm{VC} 44 \pm 16 \%$ pred). Using monodisperse aerosol particles ADAM assessed the calibres of peripheral airspaces, while $D$ measured convective gas mixing.

Among all lung function parameters, ADAM and $D$ showed the highest sensitivity and specificity for separating patients with COB from those with COB-E (area under the receiver operating characteristics curve (pROC) 0.99 and 1.0, respectively). In patients with COB aerosol parameters did not differ from those found in the control group, whereas patients with COB-E exhibited a two-fold increase in peripheral airspace dimensions compared with subjects with $\mathrm{COB}(0.86 \pm 0.07$ versus $0.37 \pm 0.02 \mathrm{~mm}$, $\mathrm{p}=0.0001)$ and an increase in $D$ by $>50 \%\left(541 \pm 74\right.$ versus $\left.345 \pm 42 \mathrm{~cm}^{3}, \mathrm{p}=0.0001\right)$.

In conclusion, aerosol-derived airway morphometry and aerosol bolus dispersion are powerful tools in the differential diagnosis of chronic obstructive pulmonary disease. Eur Respir J 1998; 12: 793-798.

One of the challenges facing medical diagnosis has been the in vivo detection of lung emphysema, which is a major component of chronic obstructive pulmonary disease (COPD) [1, 2]. However, the largest subset of patients with COPD have features of both chronic bronchitis and emphysema [3]. Chronic bronchitis and emphysema may be difficult to distinguish clinically and both may even occur without airflow obstruction [3]. Chronic bronchitis is defined on a clinical basis by chronic sputum production. Emphysema is defined anatomically by abnormal enlargement of airspaces beyond the terminal bronchioles and destruction of their walls [4].

At present, high-resolution computed tomography of the chest (HRCT) is the only noninvasive method available for reliable detection of pulmonary emphysema in vivo [5]. However, this method is expensive and logistically difficult to use in field studies. In addition, it is associated with a considerable radiation dose for the patients. Therefore, HRCT measurements are not suitable for epidemiological or occupational studies.

Using monodisperse aerosol particles, aerosol-derived airway morphometry (ADAM) assesses the calibres of airspaces, while aerosol bolus dispersion $(D)$ measures convective gas mixing. Recently, several studies have shown that the results of ADAM and $D$ are changed in patients or
*Clinic for Respiratory Medicine and **Clinical Research Group "Aerosols in Medicine" of the GSF-Institute for Inhalation Biology, Gauting, Germany

Correspondence: M. Kohlhäufl

Fachklinik München-Gauting

Zentrum für Pneumologie und Thoraxchirurgie

Robert-Koch-Allee 2

D-82131 Gauting

Germany

Fax: 498989323711

Keywords: Aerosol

aerosol bolus dispersion

airway morphometry

chronic obstructive pulmonary disease

convective gas mixing

Received: May 261997

Accepted after revision May 81998

Supported by BMBF under contract 01 GB 9504 animal models with lung emphysema [6-10]. However, these studies do not answer the question of whether, in patients with chronic bronchitis with different degrees of airflow limitation, ADAM and $D$ are able to distinguish between patients with and without emphysema. Nothing is known about the sensitivity and specificity of these techniques compared with conventional lung function parameters.

Therefore, in this study 39 patients with chronic bronchitis (19 patients with normal HRCT and 20 patients with HRCT-confirmed emphysema) were studied in order to investigate whether patients with and without emphysema can be distinguished by ADAM and $D$. The sensitivity and specificity of these tests were assessed in comparison to conventional lung function parameters. The data for the healthy control group were recently published in this journal $[11,12]$.

\section{Methods}

\section{Aerosol-derived airway morphometry}

In brief, a single breath of a monodisperse aerosol with particles of about $1 \mu \mathrm{m}$ is inhaled by the patients $[11,13]$ 
and the particle number concentration is continuously measured at the mouth as a function of the respired air volume. During postinspiratory breath-holding periods, particles slowly settle in the airspaces with a constant velocity. The smaller the airspaces, the larger the probability that particles are deposited onto airspace walls and are, therefore, not recovered upon expiration. Hence, for a given breathholding period more particles are recovered with the expired air from a larger airspace than from a smaller airspace. Moreover, the number of particles lost with increasing breath-holding periods is lower in larger airspaces than in smaller airspaces, so that the decline in the particle recovery with increasing breath-holding periods allows estimation of the airspace size [11].

The inspired aerosol can be considered to be composed of infinitesimally small volume elements, which penetrate into different volumetric lung depths $(\mathrm{Vp})$ and hence reach larger or smaller airspaces. For each of these volume elements, particle recovery decreases with increasing breathholding time. From the slope of this relation effective airspace dimensions (EAD) can be calculated for each of these volume elements, i.e. as a function of volumetric lung depth [11]. For peripheral lung regions EAD can be considered to be equivalent to the mean linear intercept [14].

To compare EAD among subjects with different lung size, inspiration was always performed up to $85 \%$ of total lung capacity (TLC). Furthermore, the Vp was normalized to the end-inspiratory lung volume ( $V \mathrm{EI}=85 \% \mathrm{TLC}$ ), so that all subjects had the same level of lung inflation, when EAD measurements were performed: relative $\mathrm{Vp}(\mathrm{Vp}, \mathrm{r})=$ $\mathrm{Vp} / \mathrm{VEI}$, so that an EAD for a given $\mathrm{Vp}$, $r$ is independent of the individual lung size and relates to comparable anatomical regions [15]. In this study, data obtained for $\mathrm{Vp}, \mathrm{r}=$ $0.14(819 \pm 95 \mathrm{~mL})$ were taken into consideration because this lung depth certainly represents peripheral lung regions and in this study all patients with COPD were able to respire the volume of air necessary for its determination.

\section{Aerosol bolus dispersion}

Since particles with diameters of about $1 \mu \mathrm{m}$ have low intrinsic mobility (i.e. diffusion or sedimentation), these particles can be used to probe intrapulmonary convective gas transport $[7,8,10,12,16,17]$. To study $D$ a subject inhales a small volume (bolus) of a monodisperse aerosol into a certain Vp and then immediately exhales. During this procedure particle number concentration is measured at the mouth as a function of the respired volume. During inspiration the bolus is supposed to divide at each airway bifurcation. At end-inspiration these sub-boluses are distributed throughout the lung. During expiration, all sub-boluses move toward the mouth and recombine at the bifurcations. Since recombination of the bolus is not completely reversible, the exhaled particles are distributed over a larger air volume than in the inspired air. Therefore, a broadened bolus is recovered from the lungs; i.e. the bolus is dispersed. To quantify $D$, the volumetric widths of the inhaled $(\mathrm{Hi})$ and exhaled bolus $(\mathrm{He})$ are quantified by the exhaled volume where particle concentration exceeds halfmaximum particle concentration (half-width: H50,i and $H 50$,e). The increase in volumetric bolus width during respiration is $D$. Bolus dispersion is determined from $H 50$,e by correcting for the bolus width of the inspired bolus $(H 50, \mathrm{i})$ :

$$
D=\sqrt{H 50, \mathrm{e}^{2}-H 50, \mathrm{i}^{2}}
$$

\section{Instrumental set-up and inhalation protocol}

ADAM and $D$ measurements were performed using the respiratory aerosol probe (Pari, Starnberg, Germany) [11, 12]. This computer-controlled device combines laser aerosol photometry with pneumotachography in order to measure the numeric concentration of respired monodisperse aerosol particles as a function of the respired air volume. Aerosol application was provided by a system of pneumatic valves, which allowed the inhalation channel to be switched between particle-free air and an aerosol supply.

All ADAM and $D$ measurements were performed at a constant airflow of $250 \mathrm{~mL} \cdot \mathrm{s}^{-1}$, controlled by use of a visual flow signal. The breathing manoeuvre for the determination of EAD started with an exhalation of half the expiratory reserve volume (ERV) followed by an inhalation of test aerosol up to $85 \%$ of TLC. After a predetermined breath-holding time, exhalation was performed until the residual volume (RV) was reached. The breathing manoeuvre was repeated for breath-holding periods of 2, 4, 6, 8 and $10 \mathrm{~s}$ duration. The breathing manoeuvre for the measurement of $D$ started from functional residual capacity (FRC) and the subjects inhaled particle-free air until the lung volume reached $85 \%$ TLC. During inspiration an aerosol bolus with $25 \mathrm{~cm}^{3}$ width was applied into a $\mathrm{Vp}$ of $400 \mathrm{~cm}^{3}$ (D400). The subjects then immediately exhaled until the entire aerosol bolus was recovered from the lungs or RV was reached. The lung depth of $400 \mathrm{~cm}^{3}$ was chosen because even in patients with emphysema, boluses inhaled into this lung depth can be completely recovered from the lungs.

\section{Particle production and classification}

Monodisperse di-2-ethylhexylsebacate (DEHS) droplets suspended in nitrogen were produced by heterogeneous nucleation of DEHS vapour on $\mathrm{NaCl}$ nuclei. The aerosol was then diluted with particle-free air to obtain a particle number concentration of about $2 \times 10^{4} \mathrm{~cm}^{-3}$. The size of the particles was classified by measuring the terminal settling velocity of the particles in a convention free sedimentation cell. The average settling velocity (mean \pm sD) of the particles throughout the study was $25 \pm 3 \mu \mathrm{m} \cdot \mathrm{s}^{-1}$, representing a mean geometrical particle diameter of $0.9 \pm 0.07 \mu \mathrm{m}$.

\section{Pulmonary function testing}

Body plethysmography and spirometry were performed using a Jäger-Masterlab (Erich Jäger, Würzburg, Germany). The following parameters were measured: TLC, VC, thoracic gas volume (TGV), RV, airway resistance (Raw), peak expiratory flow (PEF), forced expiratory volume in one second (FEV1), and maximal expiratory flows at 25, 50 and $75 \%$ of VC (MEF25, MEF50 and MEF75, respectively). Relative values of conventional lung function parameters were calculated by normalization to the reference 
values proposed by the European Community for Steel and Coal [18]. Carbon monoxide labelled with the stable oxygen isotope ${ }^{18} \mathrm{O}$ was measured as a function of the respired volume by a fast respiratory mass spectrometer (DLT 100R, improved to fit scientific standard; Wagner, Worpswede, Germany). The transfer factor of the lung for carbon monoxide $(T \mathrm{~L}, \mathrm{CO})$ was calculated as proposed by COTES et al. [19].

\section{Subjects}

Thirty-nine patients with chronic bronchitis participated in this study. Chronic bronchitis was defined as cough and sputum production occurring on most days of the month for at least 3 months $\cdot \mathrm{yr}^{-1}$ during the 2 yrs prior to the study [4]. Most of the patients were recruited during routine clinical work-up for a nonobstructing peripheral lung nodule. Pulmonary emphysema was assessed by visual assessment of inspiratory HRCT examination of the chest by an expert chest radiologist, who was unaware of the clinical and lung function data, as described by GODDARD et al. [20]. The diagnostic information obtained from direct visual observation was complemented by density analysis of the lung from HRCT slices ( $1 \mathrm{~mm}$ collimation). A set of scan images consisted of nine HRCT slices from the sternoclavicular joint down to the bottom of the lungs. Visual examination of the HRCT of all patients with chronic obstructive bronchitis (COB) and emphysema (group COB-E, $n=20$ ) showed low-attenuation areas with alteration of pulmonary parenchyma and vasculature.

The density mask technique was used to assess lung areas with attenuation coefficients lower than the inspiratory threshold of -950 Hounsfield units (HU). This is considered as a valid index of macroscopic pulmonary emphysema [21]. Patients with chronic bronchitis without macroscopic emphysema (group COB, $n=19$ ) were only accepted for recruitment if the HRCT was normal by visual examination and density analysis (range 700-850 HU). Anamnestic data were collected using a questionnaire based on American Thoracic Society (ATS) recommendations [22]. The smoking history of the patients was quantified using the cumulative cigarette consumption expressed in pack-yrs (PY). None of the patients had a history of asth-ma or allergic rhinitis. Ten subjects with COB had fixed airway obstruction and seven subjects with $\mathrm{COB}$ had rev-ersible airflow limitation, which was defined as a FEV $1<80 \%$ predicted, with a reversibility of $>15 \%$ of baseline after inhalation of $200 \mu \mathrm{g}$ salbutamol. Two patients with chronic bronchitis had a normal FEV1. Seven of the 19 patients with COB were receiving regular therapy with in-haled $\beta$-adrenergics and five were receiving twice-daily doses of theophylline. They had not received glucocorticoids or antibiotics during the preceding month, or bronchodilators within the previous $48 \mathrm{~h}$ of the study. The 20 patients with COB-E (including three subjects with $\alpha_{1}$ - antitrypsin deficiency) showed evidence of chronic irreversible hyperinflation assessed by repeated routine lung function testing and mild-to-moderate gas exchange disturbances reflected in decreased $T \mathrm{~L}, \mathrm{CO}$, decreased arterial oxygen tension $\left(\mathrm{Pa}_{\mathrm{a}} \mathrm{O}_{2}\right)$ and normal arterial carbon dioxide tension $\left(\mathrm{Pa}_{\mathrm{a}} \mathrm{CO}_{2}\right)$ (table 1$)$. Ten of the 20 patients with COB- E were receiving regular therapy with inhaled $\beta$-adrenergics and glucocorticoids and 10 were
Table 1. - Characteristics of the study population

\begin{tabular}{lcccc}
\hline & COB & COB-E & p-value & pROC \\
\hline Subjects n & 19 & 20 & & \\
Age yrs & $56 \pm 8$ & $65 \pm 7$ & 0.001 & \\
Height cm & $173 \pm 8$ & $172 \pm 10$ & Ns & \\
Sex M/F & $13 / 6$ & $17 / 3$ & & \\
Smoker/nonsmoker & $15 / 4$ & $18 / 2$ & & \\
PY & $36 \pm 3$ & $52 \pm 32$ & Ns & \\
$P$ a, ${ }_{2}$ mmHg & $76 \pm 7$ & $64 \pm 10$ & 0.0001 & \\
$P$ a,CO $\mathrm{mmHg}$ & $36 \pm 3$ & $36 \pm 5$ & Ns & \\
TLC \% pred & $99 \pm 12$ & $113 \pm 19$ & 0.01 & 0.73 \\
RV \% TLC & $40 \pm 7$ & $51 \pm 11$ & 0.0009 & 0.79 \\
TGV \% pred & $124 \pm 33$ & $157 \pm 40$ & 0.0001 & 0.84 \\
VC \% pred & $95 \pm 15$ & $95 \pm 22$ & Ns & 0.43 \\
$R$ aw kPa.L-1.- $\mathrm{s}^{-1}$ & $0.30 \pm 0.15$ & $0.41 \pm 0.27$ & Ns & 0.63 \\
FEV1 \% pred & $80 \pm 16$ & $53 \pm 21$ & 0.0001 & 0.81 \\
FEV1/VC \% pred & $66 \pm 12$ & $44 \pm 16$ & 0.0001 & 0.84 \\
MEF75 \% pred & $80 \pm 29$ & $31 \pm 27$ & 0.0001 & 0.88 \\
MEF50 \% pred & $62 \pm 26$ & $21 \pm 17$ & 0.0001 & 0.89 \\
MEF25 \% pred & $47 \pm 16$ & $29 \pm 27$ & 0.0001 & 0.87 \\
PEF \% pred & $86 \pm 22$ & $65 \pm 31$ & Ns & 0.68 \\
$T L, C O \%$ pred & $111 \pm 21$ & $73 \pm 18$ & 0.0001 & 0.88 \\
EAD mm & $0.37 \pm 0.02$ & $0.86 \pm 0.07$ & 0.0001 & 0.99 \\
$D 400 ~ \mathrm{~cm}^{3}$ & $345 \pm 42$ & $541 \pm 74$ & 0.0001 & 1.00 \\
\hline
\end{tabular}

Relative values of lung function parameters are given as a percentage of the predicted value (\% pred) [18]. Values are presented as mean $\pm S D$. COB: patients with chronic bronchitis and normal computed tomographic (CT) scan of the chest; COB-E: patients with chronic bronchitis and CT-confirmed pulmonary emphysema; pROC: $p$-value of the receiver operating characteristics [23]; M: male; F: female; PY: pack-yrs; $\mathrm{Pa}_{\mathrm{a}, \mathrm{O}_{2}}$ : arterial oxygen tension; $P \mathrm{a}_{1} \mathrm{CO}_{2}$ : arterial carbon dioxide tension; TLC: total lung capacity; RV: residual volume; TGV: thoracic gas volume; VC: vital capacity; Raw: airway resistance; $\mathrm{FEV}_{1}$ : forced expiratory volume in one second; MEF25, MEF50, MEF75: maximal expiratory flow at 25,50 and $75 \%$ of $\mathrm{VC}$, respectively; PEF: peak expiratory flow; $T \mathrm{~L}, \mathrm{CO}$ : transfer factor of the lung for carbon monoxide; EAD: effective peripheral airspace dimension; D400: aerosol bolus dispersion at a lung depth of $400 \mathrm{~cm}^{3}$. (1 $\mathrm{mmHg}=0.133 \mathrm{kPa}$.)

receiving twice-daily doses of theophylline. Arterial blood gases were measured in all patients under resting conditions.

Informed written consent was obtained from each subject. The protocol was approved by the Ethics Committee of the Medical School of the Ludwig-Maximilians-University (Munich, Germany). Results obtained in these patients were compared with previously published data from 79 healthy subjects $[11,12]$.

\section{Data evaluation}

All statistical calculations were performed using the SAS software package (SAS Institute Inc., Cary, NC, USA). The significance of differences between group averages was tested using the t-test for independent samples (SAS procedure TTEST). The requested level of significance was 0.05 . To evaluate the diagnostic value of aerosol techniques the sensitivity and specificity were quantified using the receiver operating characteristics (ROC) [23]. The area under the ROC curve represents the probability (pROC) that a randomly selected pair of patients with COB 
and COB-E is ranked correctly [24]. A pROC value of 1.0 represents maximum sensitivity and specificity.

\section{Results}

Anthropometric and lung function data of the study groups are given in table 1. Patients with COB-E were significantly older than patients with $\mathrm{COB}$. The two groups did not differ significantly in their body height, lifetime cigarette consumption, Raw, PEF (\% pred) or $\mathrm{Pa}, \mathrm{CO}_{2}$. The severity of airflow limitation based on values of FEV $1(\%$ pred) was mild-to-moderate in the group of patients with $\mathrm{COB}$ and moderate-to-severe in the group of patients with COB-E, according to the guidelines of the European Respiratory Society [4].

\section{Aerosol-derived airway morphometry}

The peripheral EAD at a $\mathrm{VP}=0.14$ in patients with $\mathrm{COB}-$ $\mathrm{E}$ was increased by more than a factor of 2 compared with patients with $\mathrm{COB}(0.86 \pm 0.07$ versus $0.37 \pm 0.02 \mathrm{~mm}, \mathrm{p}=$ $0.0001)$ or healthy subjects $(0.34 \pm 0.05 \mathrm{~mm})$ (fig. 1$)$. The peripheral EAD of patients with $\mathrm{COB}$ did not differ significantly from that measured in healthy subjects $(0.34 \pm$ $0.05 \mathrm{~mm}$ ) [11].
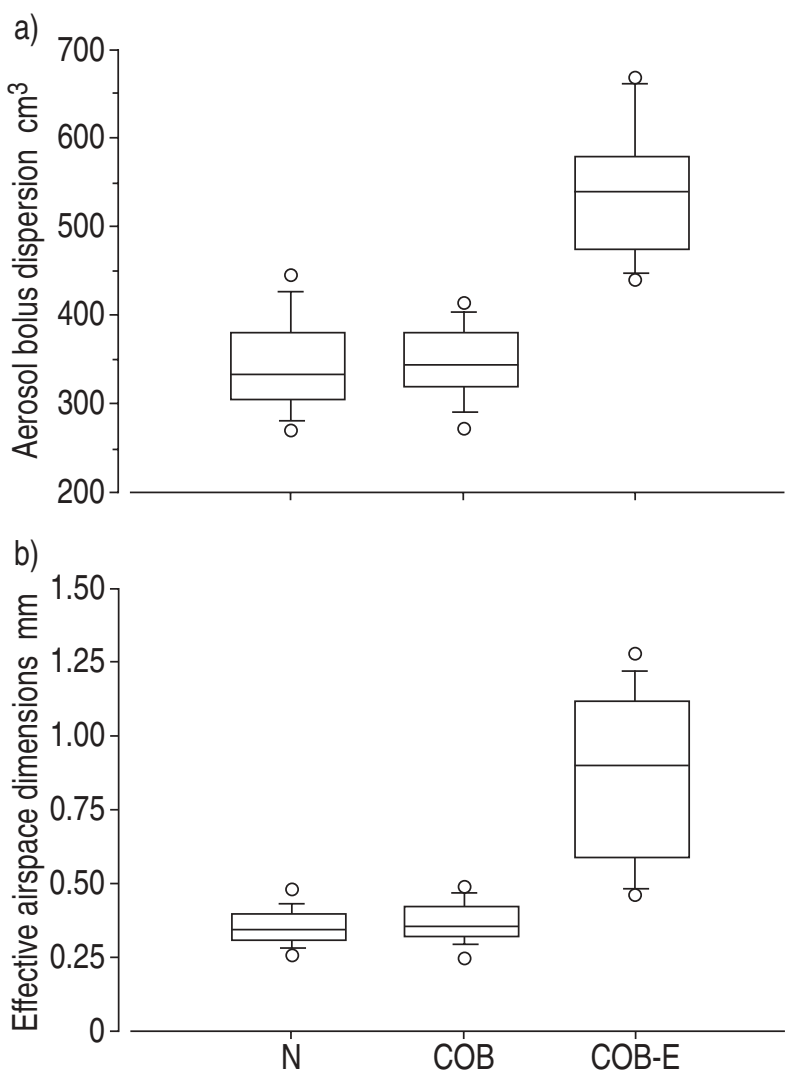

Fig. 1. - a) Aerosol bolus dispersion at a volumetric lung depth of 400 $\mathrm{cm}^{3}$ and $\mathrm{b}$ ) effective peripheral airspace dimensions for healthy subjects (N) $[11,12]$, patients with chronic bronchitis (COB) and patients with chronic bronchitis and pulmonary emphysema (COB-E) (Box-andWhisker graphics).

\section{Aerosol bolus dispersion}

Patients with COB-E showed a significant higher $D$ than patients with $\mathrm{COB}\left(541 \pm 74\right.$ versus $345 \pm 42 \mathrm{~cm}^{3}$, $\mathrm{p}=0.0001$ ) and healthy subjects [12] (fig. 1). $D$ of patients with COB did not differ significantly from that measured in healthy subjects $\left(346 \pm 53 \mathrm{~cm}^{3}\right)$ [12].

\section{Sensitivity and specificity}

Table 1 shows proc for EAD, $D$ and conventional lung function parameters for the discrimination between patients with $\mathrm{COB}$ and COB-E. $D$ showed the highest value (1.00), followed by EAD (0.99). Of the conventional lung function tests, the most sensitive were MEF50, MEF75, and TL,CO with pROC-values between 0.88 and 0.89 .

\section{Discussion}

\section{Clinical aspects}

Patients with chronic bronchitis and macroscopic emphysema (group COB-E) showed considerably increased peripheral airspace dimensions (EAD) and increased $D$, whereas in the absence of macroscopic emphysema EAD and $D$ were within normal limits. Of all the lung function parameters under consideration, EAD and $D$ had the highest sensitivity and specificity for the discrimination of patients with $\mathrm{COB}$ from those also suffering from macroscopic pulmonary emphysema (COB-E). Both parameters had pROC-values between 0.99 and 1, indicating that these tests had almost the same sensitivity and specificity as HRCT for the detection of emphysema in these study groups. All patients were able to complete the inhalation protocol. The total time required for testing was about 15 $\min$.

Previous studies indicate that EAD reflects airspace dimensions in the human lung periphery. BRAND et al. [11] showed that the peripheral EAD measured in 79 healthy subjects was similar to that measured by W.M. Thurlbeck using histological techniques and that in both studies airspace dimensions increased with age to the same extent. However, according to BRAND et al. [11], age-related differences in EAD between the study groups are neglig- ible and cannot explain the results of this study. NiKIForov et al. [25] showed a close correlation between EAD and mean linear intercept derived histologically in autopsy lungs. Increased peripheral airspace dimensions were ob-served in patients with emphysema [6]. In lungs with inhomogeneously distributed emphysematous lung injury EAD is supposed to represent a volume weighted average of airspace calibre of all ventilated lung regions [26]. EAD in patients with macroscopic emphysema showed a higher standard deviation of the mean value than in pa-tients with COB (sD 0.07 versus $0.02 \mathrm{~mm}$, respectively). This suggests a high variability in the airspace dimensions present in these patients. In patients with COB the presence of macroscopic emphysema could reliably be exclu-ded by measurement of normal peripheral EAD.

$D$ has been shown to be increased in patients with cystic fibrosis [27], in patients with lung emphysema [7] and 


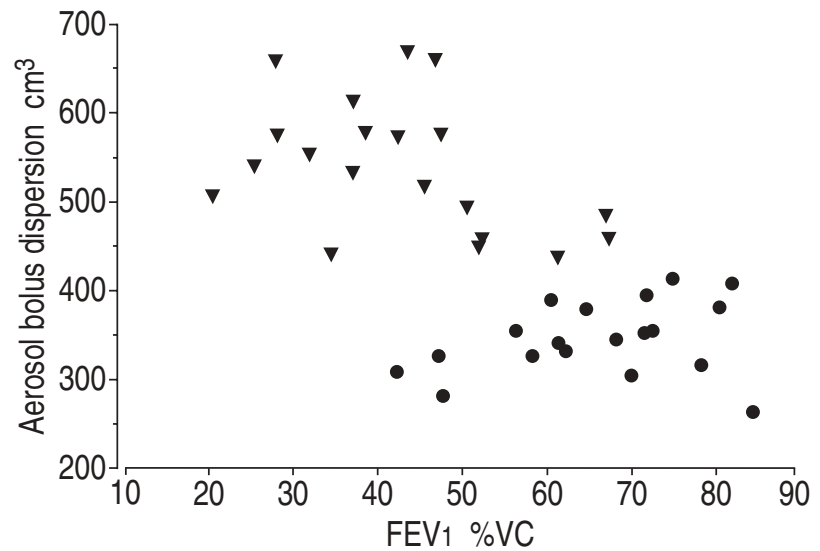

Fig. 2. - Aerosol bolus dispersion at a volumetric lung depth of 400 $\mathrm{cm}^{3}$ as a function of airflow limitation (forced expiratory volume in one second (FEV1) \% vital capacity (VC) in patients with chronic bronchitis $(\bullet)$ and patients with chronic bronchitis and pulmonary emphysema ( $\nabla)$.

in animal models with papain-induced lung emphysema [9]. It has been pointed out that $D$ increases owing to airway obstruction $[10,27]$. One of the most striking features of the data presented here is that the difference in $D$ between patients with and without emphysema is independent of the degree of airflow limitation. Figure 2 shows that subjects with COB-E have a higher $D$ than subjects with COB, even if FEV1 (\%VC) is the same. Thus, it appears that bolus dispersion is a marker for ventilation inhomogeneities caused by emphysematous lung injury.

\section{Pathophysiological aspects}

The pathophysiological mechanisms resulting in increased $D$ in patients with emphysema are still under discussion [7]. However, looking at the pathophysiological differences between $\mathrm{COB}$ with and without macroscopic emphysema may help to identity possible reasons for increased bolus dispersion. Three mechanisms may cause increased bolus dispersion: 1) central airway obstruction; 2) peripheral airway obstruction due to small airway disease; and 3 ) in patients with COB-E, morphological changes in the alveolar region with loss of alveolar attachments (radial traction) to the bronchioles.

It had been proposed that large airway changes (e.g. bronchospasm, mucosal oedema and accumulated secretions) may result in increased aerosol bolus dispersion due to turbulent airflow [27, 28]. However, despite inflammation and mucous gland hypertrophy in central airways this is generally thought not to lead to much increase in airflow resistance. Since patients with COB showed normal aerosol dispersion regardless of the degree of airflow limitation, it may be concluded that central airway constriction is not a major source of increased bolus dispersion in these patients.

Peripheral airway obstruction due to small airway disease may increase the inhomogeneity of ventilation time constants among parallel-arranged lung units [29, 30]. Ventilation time constants are given by the product of resistance and compliance of each of these compartments. Compartments with peripheral airway obstruction fill and empty with a delayed time course owing to an increased local resistance. This produces pressure differences between lung units, resulting in convective transport termed "Pendelluft". Pendelluft is supposed to increase $D[7,8,31]$. Despite clinical signs of small airway obstruction, patients with $\mathrm{COB}$ do not show an increase in bolus dispersion. Hence, increased peripheral resistance appears to be of minor importance as a determinant of bolus dispersion for the breathing pattern applied in this study.

The main histopathological difference between patients with COB-E and those with COB is the presence of morphological changes in the alveolar region in patients with COB-E, which cause loss of alveolar attachments (radial traction) to the bronchioles. The functional consequences of emphysematous lung injury are ventilation inhomogeneities due to changes in local lung compliance, peripheral expiratory airway closure and enhanced collateral ventilation between adjacent bronchopulmonary compartments [2, 32, 33]. Each of these pathophysiological mechanisms may lead to a considerably delayed aerosol particle recovery and may have a marked impact on bolus dispersion. The results of the present study support this view. The high variability in bolus dispersion observed among patients with COB-E suggests that these different mechanisms operate in patients with COB-E to a varying degree from case to case. In discussing these findings one final note of caution is that the important issue of detection of early "microscopic" emphysema [34, 35] was not addressed in the current study, because there is still controversy as to whether HRCT is sufficient to exclude adequately such histologically defined alterations [21], which may be present in patients with chronic bronchitis. The alteration referred to as "microscopic emphysema" includes disruption to elastic fibres, bronchiolar and alveolar distortion and the appearance of fenestrae in alveolar walls. Further studies will be required to answer this interesting question.

In conclusion, aerosol bolus dispersion and aerosolderived airway morphometry are powerful tools for the detection of emphysematous lung injury in patients with chronic bronchitis. Since both methods are noninvasive and rapid to perform they are suitable for screening for lung emphysema in epidemiological or occupational studies.

\section{References}

1. Flenley DC. Diagnosis and follow-up of emphysema. Eur Respir J 1990; Suppl. 9: 5s-8s.

2. Thurlbeck WM. Chronic airflow obstruction. In: Thurlbeck WM, Churg AM, eds. Pathology of the Lung. New York, Thieme, 1995; pp. 739-825.

3. Snider GL. Defining chronic obstructive pulmonary disease. In: Calverly PMA, Pride NB, eds. Chronic Obstructive Pulmonary Disease. London, Chapman \& Hall, 1995; pp. $1-8$.

4. Siafakas NM, Vermiere P, Pride NB, et al. ERS - Consensus Statement. Optimal assessment and management of chronic obstructive pulmonary disease (COPD). Eur Respir $J$ 1995; 8: 1398-1420.

5. Stern EJ, Frank MS. CT of the lung in patients with pulmonary emphysema: diagnosis, quantification, and correlation with pathologic and physiologic findings. $\mathrm{Am} \mathrm{J}$ Roentgenol 1994; 162: 791-798.

6. Beinert Th, Brand P, Behr J, Vogelmeier C, Heyder J. 
Peripheral airspace dimensions in patients with COPD. Chest 1995; 108: 998-1003.

7. Kohlhäufl M, Brand P, Meyer T, et al. Aerosol bolus dispersion: a discriminator between healthy subjects and patients with emphysema. Eur J Med Res 1997; 2: 121-128.

8. Brand $\mathrm{P}$, Tuch T, Manuwald $\mathrm{O}$, et al. Detection of early lung impairment with aerosol bolus dispersion. Eur Respir J 1994; 7: 1830-1838.

9. Rosenthal FS, Wright S. Aerosol deposition and dispersion characterize lung injury in a canine model of emphysema. J Appl Physiol 1995; 78: 1585-1595.

10. McCawley M, Lippmann M. Development of an aerosol dispersion test to detect early changes in lung function. Am Ind Hyg Assoc 1988; 49: 357-366.

11. Brand P, Rieger C, Beinert T, Heyder J. Aerosol derived airway morphometry in healthy subjects. Eur Respir $J$ 1995; 8: 1639-1646.

12. Brand P, Rieger C, Schuiz H, Beinert T, Heyder J. Aerosol bolus dispersion in healthy subjects. Eur Respir $J$ 1997; 10: 460-467.

13. Heyder J. Assessment of airway geometry with inert aerosols. J Aerosol Med 1989; 2: 89-97.

14. Rosenthal FS. Aerosol recovery following breathholding derived from the distribution of chord length in pulmonary tissue. J Aerosol Sci 1989; 20: 267-277.

15. Brand P, Beinert T, Fruhmann G, Heyder J. Aerosol derived airway morphometry at different levels of lung inflation. J Aerosol Med 1993; 6: 165-176.

16. Heyder J, Blanchard JD, Feldman HA, Brain JD. Convective mixing in the human respiratory tract: estimates with aerosol boli. J Appl Physiol 1988; 64: 1273-1278.

17. Heyder J, Davies CK. The breathing of half micron aerosols. III. Dispersion of particles in the respiratory tract. $J$ Aerosol Sci 1971; 2: 437-452.

18. Quanjer PH, Tammeling GJ, Cotes JE, Pedersen OF, Peslin R, Yernault JC. Report working party. Standardization of lung function tests. European Community for Steel and Coal. Eur Respir J 1993; 6: Suppl. 16, 5-52.

19. Cotes JE, Chinn DJ, Quanjer Ph H, Roca J, Yernault J-C. Standardization of the measurement of lung function tests. Report working party standardization of lung function tests. Official statement of the European Respiratory Society. Eur Respir J 1993; 6: Suppl. 16, 41-52.

20. Goddard PR, Nicholson EM, Laszlo G, Watt I. Computed tomography in pulmonary emphysema. Clin Radiol 1982; 33: 379-387.

21. Gevenois PA, De Vuyst P, de Maertelaer V, et al. Comparison of computed density and microscopic morphometry in pulmonary emphysema. Am J Respir Crit Care Med 1996; 154: 187-192.

22. Ferris BG. Recommended respiratory disease questionaires for use with adults and children in epidemiological research. Am Rev Respir Dis 1978; 118: 7-55.

23. Hanley JA, McNeil BJ. The meaning and use of the area under a receiver operating characteristics (ROC) curve. Radiology 1982; 143: 29-36.

24. Metz CE. Basic principles of ROC analysis. Semin Nucl Med 1978; 8: 283-298.

25. Nikiforov AI, Lippmann M, Palmes FD. Validation of an in vivo aerosol probe technique by measurements of deposition and morphometry in excised human lungs. Ann Occup Hyg 1988; 32: 33-39.

26. Blanchard JD. Aerosol bolus dispersion and aerosolderived airway morphometry: assessment of lung pathology and response to therapy, part 2. J Aerosol Med 1996; 9: 453-475.

27. Anderson PJ, Blanchard JD, Brain JD, Feldman HA, McNamara JJ, Heyder J. Effect of cystic fibrosis on inhaled aerosol boluses. Am Rev Respir Dis 1989; 140: $1317-$ 1324.

28. Muir DCF. Bulk flow and diffusion in the airways of the lung. Br J Dis Chest 1966; 60: 169-176.

29. Banjerjee M, Evans J, Jaeger MJ. Uneven ventilation in smokers. Respir Physiol 1976; 27: 277-291.

30. Barter SJ, Cunningham DA, Lavender JP, Gibellino F, Connellan SJ, Pride NB. Abnormal ventilation scan in middle-aged smokers. Am Rev Respir Dis 1985; 132: $148-151$

31. Rosenthal FS. The effect of nonuniform ventilation on the dispersion of inspired aerosol boluses: a modeling study. J Aerosol Med 1993; 6: 177-197.

32. Morell NW, Wignall BK, Biggs T, Seed WA. Collateral ventilation and gas exchange in emphysema. Am J Respir Crit Care Med 1994; 150: 635-641.

33. Saetta M, Finkelstein R, Cosio MG. Morphological and cellular basis for airflow limitation in smokers. Eur Respir J 1994; 7: 1505-1515.

34. Lamb D, McLean A, Gillooly M, Warren PM, Gould GA, MacNee W. Relation between distal airspace size, bronchiolar attachments and lung function. Thorax 1993; 48: 1012-1017.

35. McLean A, Warren PM, Gillooly M, MacNee W, Lamb D. Microscopic and macroscopic measurements of emphysema: relation to carbon monoxide gas transfer. Thorax 1992; 47: 144-149. 